\title{
Structural basis for specific recognition of pre-snRNA by Gemin5
}

Cell Research (2016) 26:1353-1356. doi:10.1038/cr.2016.133; published online 11 November 2016

\section{Dear Editor,}

The survival of motor neurons (SMN) complex assembles the heptameric rings of $\mathrm{Sm}$ proteins ( $\mathrm{Sm}$ core) on small nuclear RNAs (snRNAs) to form small nuclear ribonucleoprotein particles (snRNPs) [1-4], the major components of the spliceosome [5]. The SMN complex consists of SMN, Unrip and Gemin2-8 [6, 7]. SMN deficiency leads to correspondingly reduced snRNP assembly and is an underlying cause for spinal muscular atrophy, a common motor neuron degenerative disease [8]. The SMN complex functions to ensure Sm core assembly only on the correct snRNAs, therefore preventing aberrant $\mathrm{Sm}$ core formation. Such stringent specificity of the SMN complex toward snRNAs is conferred by Gemin5, which directly interacts with snRNA precursors (pre-snRNAs) [9-11] and delivers them to sites of Sm core assembly and processing. Gemin5 recognizes the snRNP code comprised of the Sm site $\left(\mathrm{AU}_{5-6} \mathrm{G}\right)$ and an adjacent 3 '-terminal stem loop via its N-terminal WD40 repeat domain (termed Gemin5-WD). Gemin5 also has been shown to bind the 7-methylguanosine $\left(\mathrm{m}^{7} \mathrm{G}\right)$ cap [12] and to downregulate internal ribosome entry site-dependent translation [13]. Although the function of Gemin 5 in the biogenesis of snRNPs has been explored, the molecular mechanism for specific recognition of pre-snRNAs by Gemin 5 remains elusive.

To reveal the molecular basis for specific recognition of pre-snRNAs by Gemin5, we determined the crystal structures of Gemin5-WD (aa 1-740) in its apo form and in complex with an $\mathrm{m}^{7} \mathrm{GpppG}$ cap analog and the $\mathrm{Sm}$ site RNA (5'-AAUUUUUGAC-3' corresponding to nt 118-127 in pre-U4 snRNA) at resolutions of 3.0, 2.57 and $2.49 \AA$, respectively (Supplementary information, Table S1). Gemin5-WD contains 14 repeats of canonical WD40-motifs that fold into two connected seven-bladed $\beta$-propellers (Supplementary information, Figure S1A). The two doughnut-shaped propellers (WD1: blades 1-7, aa 19-377 and WD2: blades 8-14, aa 3-10 and 428-712) are similar in size, each $\sim 38 \AA$ in diameter and $\sim 55 \AA$ high, and twisted $\sim 15^{\circ}$ askew relative to one another. The extreme N-terminal region of WD1 forms the outermost $\beta$-strand of blade 14 in WD2. Such a structural motif referred to as "molecular velcro" has been observed in the structures of Aip1 (Supplementary information, Figure S1A).

The structure of the Gemin5-WD/m ${ }^{7} \mathrm{GpppG}$ complex shows that the capped guanosine, $\mathrm{m}^{7} \mathrm{G}$ is inserted into the pocket at the top surface of WD2 (Figure 1A), whereas the other guanosine is not observed in the electron density map (Supplementary information, Figure S1B). Specifically, $\mathrm{m}^{7} \mathrm{G}$ is sandwiched between the aromatic ring of Tyr474 and the side chains of Leu580 and Asn582 (Figure 1B). In addition to the stacking interactions, the main chain carbonyl groups of Thr540 and Asn582, and the side chain carboxyl group of Glu541 are hydrogen bonded to the $\mathrm{N} 1$ and $\mathrm{N} 2$ atoms of $\mathrm{m}^{7} \mathrm{G}$, respectively, whereas the side chains of Tyr474, Tyr660 and Arg684 form hydrogen bonds with the $\alpha$ - and $\gamma$-phosphate groups. Additional contacts involve the side chain of Lys641 and the ribose ring and the $\beta$-phosphate group. Consistent with the structure, mutations of invariant Tyr474, Lys641, Tyr660 and Arg684 (Supplementary information, Figure S1C) to Ala abolished the cap-binding activity of Gemin5, underscoring the importance of these residues in cap recognition (Figure 1C). The recognition of $\mathrm{m}^{7} \mathrm{G}$ by Gemin5 is specific as placing a trimethylated (Tri-mG) cap into the position occupied by the $\mathrm{m}^{7} \mathrm{G}$ cap would cause severe steric clashes with the neighboring residues (Supplementary information, Figure S1D). Gemin5-WD recognizes the $\mathrm{m}^{7} \mathrm{G}$ cap in a manner similar to those observed in the structures of $\mathrm{CBC} 20$, eIF4E, PARN and DcpS in complex with $\mathrm{m}^{7} \mathrm{GpppG}$ (Supplementary information, Figure S1E).

The structure of Gemin5-Sm RNA complex shows that the Sm site RNA binds to a positively charged concave surface formed between the two $\beta$-propellers WD1 and WD2 (Figure 1D). Gemin5-WD does not show significant conformational changes upon Sm RNA binding, as superposition of Gemin5-Sm RNA structure onto the apo-form gives a root mean-square deviation of $0.3 \AA$ (Supplementary information, Figure S1A). Out of 

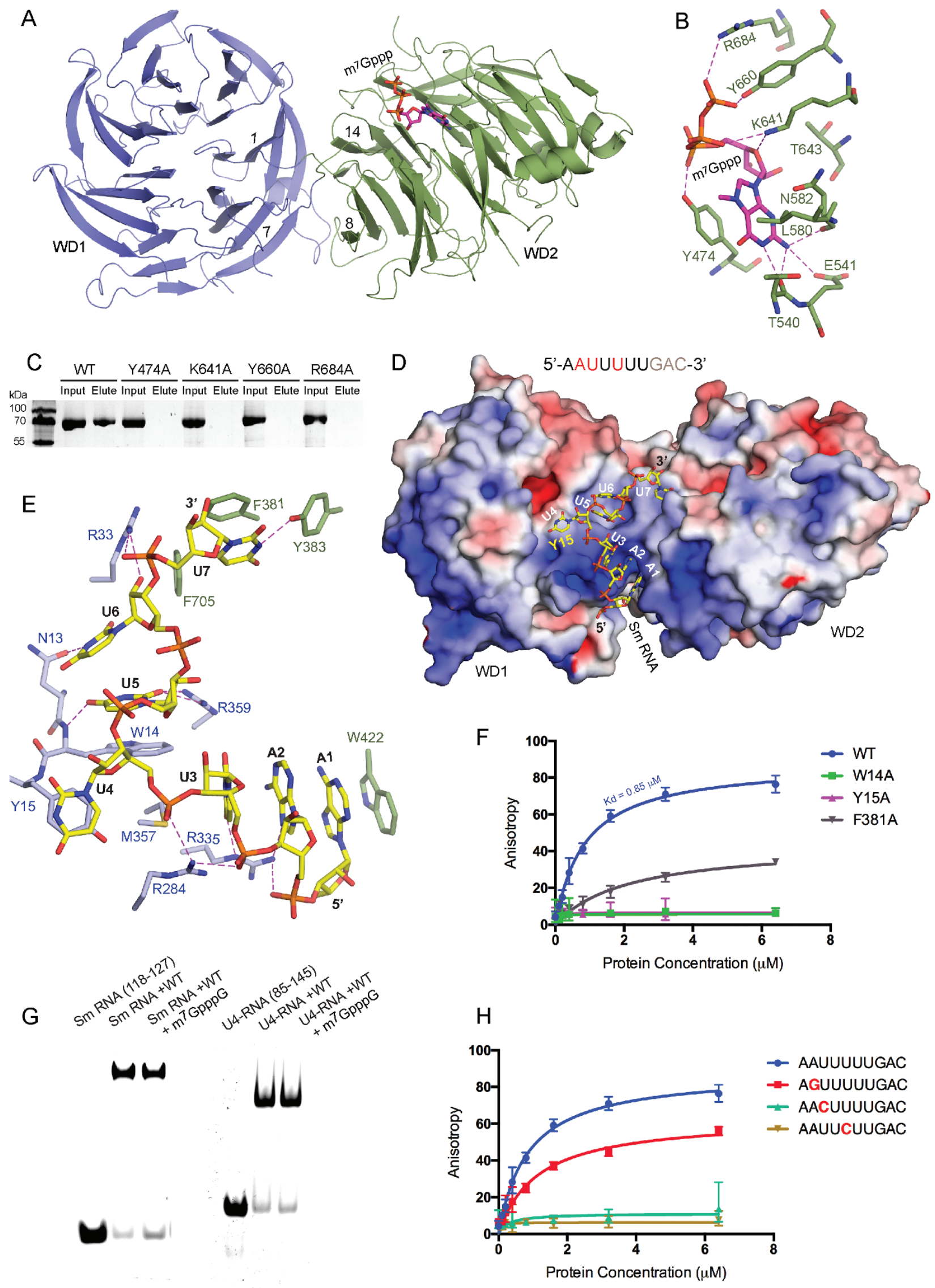

SPRINGER NATURE | Cell Research | Vol 26 No 12 | December 2016 
the 10 nucleotides used for crystallization, only 7 nucleotides are observed in the electron density map (Supplementary information, Figure S1B). The bound Sm RNA displays an extended conformation with a U-shaped bend between U3 and U5, such that the uridine base of U4 flips out, pointing in the direction opposite to those of U3 and U5. The 5' end of the Sm RNA contacts blade 8 , and the $3^{\prime}$ end contacts blade 14 with majority of the interactions involving residues from WD1.

The Sm RNA is recognized specifically through two grooves formed on either side of Tyr15 in WD1 (Figure 1D). Tyr15 stacks against a flipped nucleotide U4 in the bound RNA. Toward the $5^{\prime}$ end of U4 is a shallow groove formed between blades 7 and 8 that holds nucleotides U3 and A2. The specificity of A2 and U3 is ensured by hydrogen-bonding interactions between their bases and the guanidinium group of Arg335 (Figure 1E). U3 and A2 stack against each other as well as against the first nucleobase, A1. Trp422 belonging to blade 8 of WD2 completes the interaction through stacking against A1. Toward the 3' end of U4 is a pocket formed by residues Trp14, Arg359, Asn13 and Arg33, which holds the nucleobases U5 and U6. Trp14 stacks against U5, which in turn stacks against U6. Arg359 and the main chain amide group of Trp14 recognize the base of U5, whereas Asn13 recognizes the base of U6. The side chain of Arg33 is hydrogen-bonded to the ribose of U6 and the subsequent phosphate moiety. The terminal nucleobase of U7 stacks against Phe 381 and is surrounded by hydrophobic residues Tyr383 and Phe705 (Figure 1E). In contrast to our structure showing the specific recognition of RNA, the WD40 domain of DDB2 recognizes dsDNA in a sequence-independent manner [14] (Supplementary information, Figure S1F). These results confirm that WD40 domain is a versatile nucleic acid-binding module in addition to its role in protein-protein interactions.

To further characterize the role of residues involved in Sm RNA binding, we mutated several conserved residues (Supplementary information, Figure S1C) in Gemin5-WD and checked the mutational effects on
RNA binding (Figure 1F). Of the mutants we generated, mutation of Trp14 or Tyr15 to Ala completely abolished the RNA-binding ability of Gemin5, whereas single Ala substitution of Phe381 dramatically reduced RNA binding, underscoring the essential role of these three residues in Sm RNA binding. As Sm RNA-binding and cap-binding sites on Gemin5-WD are in close proximity, we also investigated whether Sm RNA binding-defective mutants, W14A, Y15A and F381A can bind the cap analog. As expected, these mutants showed similar binding affinity to that of the wild-type protein (Supplementary information, Figure S1G). Furthermore, RNA-binding assays in the presence of 2.5 -fold excess of $\mathrm{m}^{7} \mathrm{GpppG}$ showed that $\mathrm{m}^{7} \mathrm{GpppG}$ had no effect on Gemin5-WD's binding to RNA (Figure 1G). Taken together, these results suggest that Gemin5 recognizes the Sm site and the $\mathrm{m}^{7} \mathrm{G}$ cap of the pre-snRNA through two distinct binding sites.

Gemin5 has been shown to specifically recognize the 5'-AUUUUUG-3' Sm site sequence of HSUR5 RNA of HVS with the first adenosine and the first and third uridines (corresponding to A2, U3 and U5 in Figure $1 \mathrm{E}$ ) corresponding to the specificity determinants of pre-snRNA for Gemin5 recognition [9]. Moreover, pre-U1 snRNA containing a non-canonical $\mathrm{Sm}$ site 5'-AUUUGUG-3' shows relatively weaker affinity to Gemin5 [11]. To verify the specificity determinants, we examined Gemin5-WD binding activities towards mutated Sm RNAs (Figure 1H). Substitution of A2 to G significantly decrease the RNA binding to Gemin5-WD, whereas the RNA binding was abolished when either U3 or U5 was substituted to C. Consistent with these findings, the specificity of $\mathrm{A} 2$ and $\mathrm{U} 3$ is ensured by the hydrogen bonds from the side chain of Arg335 to the purine and pyrimidine rings of $\mathrm{A} 2$ and $\mathrm{U} 3$, respectively. The specificity of U5 is provided by the hydrogen bonds between its base and Arg359. U6 corresponds to G5 in pre-U1 snRNA; substitution of U6 to $G$ in the structure of Gemin5-Sm RNA would cause steric clashes with the main chain of Arg33 and side chain of Asn13.

Figure 1 Specific recognition of $\mathrm{m}^{7} \mathrm{GpppG}$ cap and the Sm site of pre-snRNA by Gemin5-WD. (A) Overall structure of Gemin5-WD in complex with $\mathrm{m}^{7} \mathrm{GpppG}$ cap. WD1 and WD2 of Gemin5-WD are shown in light blue and green, respectively. $\mathrm{m}^{7} \mathrm{Gppp}$ of the cap is shown in purple. (B) Interactions of the $\mathrm{m}^{7} \mathrm{GpppG}$ cap analog with Gemin5-WD. Residues involved in the interaction are shown as sticks and labeled. Hydrogen bonds are shown as pink dashed lines. (C) Cap-binding assays were performed with wild-type (WT) Gemin5-WD and its mutants. (D) Electrostatic surface potential of Gemin5-WD complexed with Sm RNA (-7.5-7.5 kT/e). Sm RNA (nt 118-127 in pre-U4) used in the study is shown with specificity-determining nucleotides in red and those not observed in the structure in grey. (E) Interactions of Sm RNA with Gemin5-WD. (F) Fluorescence anisotropy measurements of Sm RNA (nt 118-127) binding with Gemin5-WD and its variants. (G) RNA-binding assays of Gemin5-WD in the absence or the presence of 2.5-fold excess of $m^{7}$ GpppG. Sm RNA (nt 118-127) and longer U4-RNA (nt 85-145 in pre-U4) are used. (H) The specificity determinants of Sm RNA for Gemin5 recognition were examined by fluorescence anisotropy. 
Trp286 has been reported to be a key residue for the recognition of both $\mathrm{m}^{7} \mathrm{G}$ and the Sm site RNA [11, 12]. However, Trp286 is located in a hydrophobic core of blade 6 of WD1, contacting Tyr15, which stacks against the flipped nucleotide U4 (Supplementary information, Figure $\mathrm{S} 1 \mathrm{H})$. Mutation of this residue is likely to destabilize the conformation of Tyr15, therefore affecting RNA binding. Trp286 is located far away from the cap-binding site. Therefore, it is unlikely to be involved in cap binding as suggested by Bradrick and Gromeier [12]. An alternative possibility is that mutation of Trp286 would disrupt the fold of Gemin5, thereby abolishing both cap and RNA binding of Gemin5. Consistent with this notion, we found that mutation of Trp286 to Ala led to the mutant protein being expressed in the inclusion bodies and insoluble.

In summary, we report structures of the WD40 domain of Gemin 5 in complex with the $\mathrm{m}^{7} \mathrm{G}$ cap and the Sm site RNA. Gemin5 specifically recognizes the Sm RNA and the $m^{7} G$ cap through its first and second $\beta$-propellers, respectively. This dual recognition mechanism ensures the assembly of Sm core only on correct snRNAs, thereby preventing illicit $\mathrm{Sm}$ core formation. Our results provide a framework for further elucidating how Gemin 5 delivers pre-snRNAs to the SMN complex for the biogenesis of snRNPs. The atomic coordinates and structure factors for apo Gemin5-WD, Gemin5-WD/m ${ }^{7}$ GpppG and Gemin5WD/Sm RNA complex structures have been deposited into Protein Data Bank under the accession code of $5 \mathrm{H} 3 \mathrm{~S}, 5 \mathrm{H} 3 \mathrm{~T}, 5 \mathrm{H} 3 \mathrm{U}$ respectively.

\section{Acknowledgments}

We would like to thank the beamline scientists at the beamlines PX-I (SLS, PSI, Switzerland), ID-23-1 (ESRF, Grenoble, France) and BL13B1 (NSRRC, Taiwan, China) for assistance of X-ray data collection. This work was supported by the Agency for Science, Technology and Research in Singapore.

Xuhua Tang ${ }^{1}$, Sakshibeedu R Bharath ${ }^{1}$, Shunfu Piao ${ }^{1}$, Vanessa Qianmin Tan ${ }^{1}$, Matthew W Bowler ${ }^{2,3}$, Haiwei Song ${ }^{1,4}$
${ }^{I}$ Institute of Molecular and Cell Biology, 61 Biopolis Drive, Proteos, Singapore 138673, Singapore; ${ }^{2}$ European Molecular Biology Laboratory, Grenoble Outstation, 71 avenue des Martyrs, CS 90181 F-38042 Grenoble, France; ${ }^{3}$ Unit of Virus Host-Cell Interactions, Univ. Grenoble Alpes-EMBL-CNRS, 71 avenue des Martyrs, CS 90181 F-38042 Grenoble, France; ${ }^{4}$ Department of Biochemistry, National University of Singapore, 14 Science Drive, Singapore 117543, Singapore

Correspondence: Haiwei Song

E-mail: haiwei@imcb.a-star.edu.sg

\section{References}

1 Pellizzoni L, Yong J, Dreyfuss G. Science 2002; 298:1775-1779.

2 Meister G, Buhler D, Pillai R, et al. Nat Cell Biol 2001; 3:945-949.

3 Kroiss M, Schultz J, Wiesner J, et al. Proc Natl Acad Sci USA 2008; 105:10045-10050.

4 Grimm C, Chari A, Pelz JP, et al. Mol Cell 2013; 49:692-703.

5 Will CL, Luhrmann R. Spliceosomal UsnRNP biogenesis, structure and function. Curr Opin Cell Biol 2001; 13:290-301.

6 Battle DJ, Kasim M, Yong J, et al. The SMN complex: an assembly machine for RNPs. Cold Spring Harb Symp Quant Biol 2006; 71:313320 .

7 Carissimi C, Baccon J, Straccia M, et al. FEBS Lett 2005; 579:23482354.

8 Rossoll W, Bassell GJ. Results Probl Cell Differ 2009; 48:289-326.

9 Battle DJ, Lau CK, Wan L, et al. Mol Cell 2006; 23:273-279.

10 Yong J, Kasim M, Bachorik JL, et al. Mol Cell 2010; 38:551-562.

11 Lau CK, Bachorik JL, Dreyfuss G. Nat Struct Mol Biol 2009; 16:486491.

12 Bradrick SS, Gromeier M. PLoS One 2009; 4:e7030.

13 Pineiro D, Fernandez N, Ramajo J, et al. Nucleic Acids Res 2013; 41:1017-1028.

14 Scrima A, Konickova R, Czyzewski BK, et al. Cell 2008; 135:12131223.

(Supplementary information is linked to the online version of the paper on the Cell Research website.)

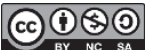

This license allows readers to copy, distribute and transmit the Contributionas long as it attributed back to the author. Readers are permitted to alter, transform or build upon the Contribution as long as the resulting work is then distributed under this is a similar license. Readers are not permitted to use the Contribution for commercial purposes. Please read the full license for further details at - http://creativecommons.org/ licenses/bync-sa/4.0/

(C) The Author(s) 2016 